\title{
Belastende Lebenserfahrungen und Therapieergebnisse: Eine explorative Studie in einer stationären Drogentherapieeinrichtung
}

Strainful Life-events and Treatment Results: An Explorative Study in an In-patient Drug Treatment Unit

\section{Zusammenfassung}

Studienziel: Es wurde die Life-time-Prävalenz von belastenden Lebensereignissen bei Teilnehmer/Innen eines stationären Drogentherapieprogramms erhoben und explorativ auf Zusammenhänge mit Therapieergebnissen überprüft. Untersuchungsmethode: $\mathrm{n}=188$ konsekutive Therapiebeender/Innen (74\% Männer; 26\% Frauen) in den Sozialtherapeutischen Wohngemeinschaften des Projekts JORK - Jugendhilfe e.V. Hamburg. Die Untersuchungsgruppe war charakterisiert durch erhebliche psychosoziale Belastungen, z.B. ein Drittel ohne Hauptschulabschluss, etwa drei Viertel ohne Berufsausbildung, im Mittel etwa dreizehnjährige Drogenabhängigkeit. Es wurden retrospektiv die Therapiedokumentationen auf das Vorhandensein von 15 belastenden Lebensereignissen ausgewertet. Als Therapieergebniskriterien wurden berücksichtigt: Therapiezeit, reguläre vs. vorzeitige Therapiebeendigung, beruflich-schulische Integration am Therapieende und Therapieverbesserungen gemäß Mitarbeiterrating. Ergebnisse: Bei den Therapieteilnehmer/Innen waren im Mittel vier belastende Lebensereignisse dokumentiert (Frauen $M=4,7$; Männer $M=3,8)$. Im Detail: Schwere körperliche Gewalterfahrungen: $56 \%$ Haftaufenthalte: $60 \%$ aufgewachsen ohne beide Elternteile: 38\% mindestens einen Suizidversuch: 35\% ein Elternteil suchtmittelabhängig: 30\% sexuelle Gewalterfahrung: Frauen 53\%, Männer 9\%. Klient/Innen ohne schwere körperliche Gewalterfahrung, ohne Hafterfahrung bzw. ohne Suizidversuch hatten eine längere Therapiezeit. In der Regressionsanalyse konnten $8 \%$ der Varianz erklärt werden $(R=0,30)$, wobei vor allem schwere körperliche Gewalterfahrung und etwas schwächer Haftaufenthalt als Einflussvariablen identifiziert wurden. Klient/ Innen ohne schwere körperliche Gewalterfahrung, ohne Hafterfahrung bzw. ohne Suizidversuch beendeten die Therapie häu-

\section{Abstract}

Object of study: Exploration of clients of a residential drug addiction treatment program with regard to stressful life events in their biography. Correlations of prevalence of stressful life events and treatment outcome were examined. Method: 188 former clients of therapeutic community "Project JORK" in Hamburg (74\% males, $26 \%$ females) were examined. The client group was characterized by considerable psychosocial stress in the biography, e.g. one third had reported that they had not finished secondary school, about three quarters had no vocational training, in average subjects had been drug dependent for some thirteen years. Treatment records were evaluated as to 15 stressful life events. Outcome criteria were: treatment time, leaving treatment prematurely vs. regular completion, educational-vocational integration after treatment, staff rating of general improvement. Results: In average four stressful life events were recorded among clients (females 4.7 , males 3.8): suffering heavy physical violence (56\%), imprisonment (60\%), being raised without parents (38\%), at least one suicidal attempt (35\%), one parent being addicted to some substance (30\%), suffering sexual assault (females $53 \%$, males $9 \%$ ). Clients who had not been exposed to heavy physical violence, had not been imprisoned and/or had no suicidal attempts stayed longer in treatment. Regression analysis explained $8 \%$ of variance $(R=0.30)$. Having suffered from heavy violence and, somewhat lower, imprisonment were identified as influential variables. More of those clients who had had not been exposed to physical violence, imprisonment and/or had no suicidal attempt completed the treatment program. Logistic regression yielded that only exposition to physical violence improved prediction of leaving treatment prematurely vs. completion of program. Altogether only 64\% could be classified correctly (Nagel- 
figer regulär. In einer logistischen Regression verbesserte nur die schwere körperliche Gewalterfahrung die Vorhersage regulärer vs. vorzeitiger Therapiebeendigung; insgesamt wurden jedoch nur 64\% richtig klassifiziert (Nagelkerkes $\mathrm{R}^{2}=0,13$ ). Klient/Innen ohne schwere körperliche Gewalterfahrung waren am Therapieende häufiger beruflich-schulisch integriert, und gemäß Mitarbeiterrating erzielten sie häufiger größere Therapieverbesserungen. Beim Vergleich zweier mediangesplitteter Extremgruppen mit weniger ( $M=1,8$; Range $0-3)$ bzw. mehr belastenden Lebensereignissen ( $M=5,6$; Range $4-10)$ hatte die weniger belastete Gruppe eine längere Therapiezeit und häufiger regulär die Therapie beendet; sie war am Therapieende häufiger in $\mathrm{Ar}-$ beit/Ausbildung integriert und hatte häufiger größere Therapieverbesserungen gemäß Mitarbeiterrating erzielt (OR: 1,9 bis 2,4). Schlussfolgerung: Um Haltequote und Therapieergebnisse zu verbessern, muss überlegt werden, wie vor allem den KlientInnen mit stärkeren biographischen Belastungen, insbesondere mit schweren körperlichen Gewalterfahrungen, adäquater geholfen werden kann.

\section{Schlüisselwörter}

Stationäre Drogentherapie · medizinische Rehabilitation · therapeutische Gemeinschaft · belastende Lebensereignisse - traumatische Belastungen kerke's $R^{2}=0.13$ ). Clients who had not been exposed to heavy physical violence were found to have more often vocational training after treatment and showed better general improvement according to staff ratings. Comparison of two extreme groups (splitted by median), one with fewer stressful life events $(A=1.8$, range $0-3)$, the other with more stressful life events $(A=5.6$, range $4-10)$ indicated that the client group with fewer stressful life events had longer treatment times and more frequently completed the treatment program. More clients of this group had jobs or vocational training after treatment and showed better improvement according to staff rating (OR: 1.9 2.4). Conclusion: In order to achieve better retention rates and treatment outcomes one should consider, how to give adequate help to clients with stronger stress experiences in their biography, in particular to those who have suffered from heavy physical violence.

Key words

Residential drug addiction treatment - medical rehabilitation . therapeutic community · stressful life events · traumatic experience

Suchtpatient/Innen schwieriger und weniger erfolgreich verläuft [10-16, 20-23]. Suchttherapieexperten schätzten die Therapie von Patienten mit gleichzeitiger Suchtmittelabhängigkeit und Traumabelastung als schwieriger ein [17]. Bei einer bundesweiten Befragung in Einrichtungen der ambulanten Suchttherapie berichteten ebenfalls viele Suchttherapeuten bei der Betreuung traumatisierter Suchtpatient/Innen über häufigere Probleme in der therapeutischen Beziehung sowie über schwierigere Therapieverläufe [19]. Hieran anknüpfend, versucht die vorliegende Studie explorativ der Frage nachzugehen, ob es in der stationären Drogentherapie Zusammenhänge zwischen schweren biographischen Belastungen und Therapieergebnissen gibt. Dabei wird generell angenommen, dass Klient/Innen mit schwer belastenden Lebensereignissen andere Therapieergebnisse erzielen als Klient/Innen ohne bzw. mit weniger belastenden Lebensereignissen.

\section{Untersuchungsbeschreibung}

In der Hamburger Basisdatendokumentation, in der knapp 12000 verschiedene, im Jahre 2004 in den ambulanten Alkohol- oder Drogenberatungsstellen betreute Personen berücksichtigt wurden, konnten bei der Hälfte der alkoholabhängigen und bei etwa zwei Drittel der drogenabhängigen Frauen und Männer Life-timePrävalenzen schwerer körperlicher Gewalterfahrungen dokumentiert werden. Etwa jede Dritte der alkoholabhängigen und die Hälfte der drogenabhängigen Frauen hatten im Leben sexuelle Gewalterfahrungen gemacht. Aber auch die 12-Monatsprävalenzwerte waren jeweils beträchtlich. Crack-Konsument/Innen waren dabei besonders belastet durch Gewalterfahrungen [9].

In der angloamerikanischen Literatur gibt es aus unterschiedlichen ambulanten und stationären Therapieprogrammen eine Fülle von Hinweisen, dass die Therapie von traumatisierten

\section{Konzeptionelle Aspekte}

$\mathrm{Zu}$ den besonderen Profilmerkmalen der Hamburger Sozialtherapeutischen Wohngemeinschaften für Drogenabhängige gehört neben der Behandlung der Suchtproblematik in kleineren therapeutischen Gemeinschaften die frühzeitige Orientierung auf die Integration in „normale“ Arbeits- oder Ausbildungsverhältnisse als ein besonderer Schwerpunkt der Rehabilitation (www. projekt-jork.de).

Im Untersuchungszeitraum war die auf in der Regel 10 Monate konzipierte Gesamttherapie strukturell untergliedert in eine Entwöhnungsphase von vier bis sechs Monaten und eine interne Adaptionsphase von ebenfalls vier bis sechs Monaten Dauer. Der 
Aufenthalt in den jeweiligen Abschnitten der Rehabilitation richtete sich nach den individuellen Eingangsvoraussetzungen und dem Therapieverlauf.

In der Entwöhnungsphase fand ein strukturiertes Wochenprogramm mit personen- und themenzentrierten Gruppengesprächen, Einzelgesprächen und -beratung, gesundheitsfördernden, allgemeinbildenden und interessenweckenden Gruppenangeboten, Hausdiensten, Freizeitaktivitäten sowie mit individuell vereinbarten Aufgaben statt.

Die zielstrebige Auseinandersetzung mit der beruflich-schulischen Perspektive erfolgte von Beginn an in einer Berufsorientierungsgruppe, über Einzelberatung und systematische Einzelfallhilfe.

Bedingung für den Wechsel in die interne Adaptionsphase war neben dem Erreichen anderer Therapieziele - das Vorhandensein eines zumindest in absehbarer Zeit extern antretbaren Schul-, Ausbildungs-, Praktikums- oder Arbeitsplatzes.

In der Adaptionsphase stand der Einstieg in Arbeit/Ausbildung als Arbeits- und Belastungserprobung im Mittelpunkt. Die Bewältigung dieses für die meisten Klienten großen Schrittes wurde sozialpädagogisch begleitet. Zusätzlich nahmen die Klienten nachmittags, abends und am Wochenende am therapeutischen Programm teil $[23,24]$.

Im Untersuchungszeitraum unterhielt die Einrichtung 44 Therapieplätze in fünf therapeutischen Gemeinschaften am Rande Hamburgs mit Anbindung an das öffentliche Verkehrsnetz. Die sozialtherapeutische Gruppenbetreuung erfolgte bei einem Betreuungsschlüssel von etwa 1:3 vorwiegend durch Sozialpädagog/Innen.

\section{Datenerhebung}

Bei der vorliegenden Untersuchung handelt es sich um eine explorative naturalistische Studie mit retrospektiver Datenerhebung: Die Untersuchungsgruppe wurde nicht kontrolliert oder randomisiert zusammengestellt, vielmehr wurden alle konsekutiven Therapiebeender vom 1.1.2002 - 31.3.2004 einbezogen. Die maximale Größe der Untersuchungsgruppe betrug $n=188$. Die Aufnahme in die Einrichtung und die Durchführung der stationären Rehabilitation erfolgten nicht „standardisiert“, sondern so, wie es konzeptionell vorgesehen war. Die Erhebungsinstrumente und der Zeitpunkt der Datenerhebung waren durch das Selbstevaluationssystem der Einrichtung festgelegt [6].

\section{Erhebung belastender Lebensereignisse}

Anhand eines Auswertungsrasters wurden von einem Diplomanden alle Therapiedokumentationen (Aufnahmedokumentation, Lebensläufe, Protokolle) überprüft. Es wurde jeweils registriert, wenn eines der folgenden, als in der Regel belastend definierten 15 Lebensereignisse erwähnt wurde:

1. Vater bzw. Eltern unbekannt

2. aufgewachsen mit nur einem Elternteil/ohne Eltern

3. Trennung der Eltern bis 16. Lebensjahr

4. mindestens ein Elternteil suchtmittelabhängig

5. Heimaufenthalte/öffentliche Erziehung

6. häufiger Wechsel des Wohnortes in Kindheit/Jugend
7. Tod enger Bezugspersonen

8. schwere körperliche Gewalterfahrungen

9. sexuelle Gewalterfahrungen

10. Suizidversuche

11. Haftaufenthalt

12. Psychiatrieaufenthalte

13. Prostitution

14. eigene schwere Gewaltausübung

15. andere belastende Erfahrungen (u.a. Unfälle, schwere Erkrankungen, Miterleben von Gewalttaten).

\section{Ergebniskriterien}

Folgende Therapieergebniskriterien wurden berücksichtigt:

1. Therapiezeit: Die mittlere Therapiezeit aller Klient/Innen lag bei 165 Tagen $(s=123)$, der Median bei 142 Tagen. Die Daten wurden aus der Therapiedokumentation übernommen.

2. Art der Therapiebeendigung (regulär vs. vorzeitig): Die Art der Therapiebeendigung wurde im Therapieabschlussbogen als Teil des Selbstevaluationssystems festgehalten [6]. 45\% hatten die stationäre Therapie regulär beendet (mittlere Therapiezeit $M=279$ Tage; $s=86 ; n=85$ ), $55 \%$ vorzeitig durch „Abbruch“ oder Entlassung (mittlere Therapiezeit $M=72$ Tage; $s=47 ; n=103$ ). „Reguläre Beendigung“ bedeutete, dass am Ende der vorgesehenen Therapiezeit der Auszug planmäßig und vorbereitet, in der Regel in eigenen Wohnraum, erfolgte.

3. Integration in Arbeit/Ausbildung bei Therapiebeendigung (integriert vs. nicht integriert): Die Ergebnisse der beruflichschulischen Integration wurden ebenfalls im Therapieabschlussbogen festgehalten [6]. 46\% $(n=75)$ aller Klient/Innen waren bei Therapiebeendigung beruflich-schulisch integriert, im Mittel seit fast vier Monaten ( $M=3,7 ; s=3,0)$. Dies war von der Konzeption her in der Regel erst mit Beginn der Adaptionsphase möglich. Diese Teilgruppe der beruflichschulisch Integrierten setzte sich wie folgt zusammen: $4 \%$ besuchten eine Schule zwecks Erreichung des Haupt- oder Realschulabschlusses, 9\% absolvierten eine Berufsausbildung, Umschulung, oder sie besuchten eine berufsbildende Fach- oder Hochschule; $5 \%$ arbeiteten bei einer Hamburger Beschäftigungsgesellschaft, 15\% standen in einem Arbeitsverhältnis im ersten Arbeitsmarkt und 1\% war selbstständig tätig; 6\% nahmen an einem Praktikum teil, weitere 6\% an einem qualifizierenden Kursus; 54\% $(n=88)$ waren bei Therapiebeendigung nicht integriert. Durch Missings lagen in dieser Variablen Daten für $n=163$ vor.

4. Therapieverbesserungen aus Mitarbeitersicht (größere vs. geringere): Bei Therapiebeendigung schätzte der für den jeweiligen Klienten zuständige Mitarbeiter im Therapieabschlussbogen [6] die während der Therapie eingetretenen Verbesserungen in den Bereichen „Gesundheit“, „Schulischberufliche Situation“, „Soziale Situation“, „Freizeitverhalten“, „Wohnsituation“ und „Persönlichkeitsstabilisierung“ anhand fünfstufiger Ratingskalen (Pole: „nicht verbessert“ und "sehr deutlich verbessert“) ein. Die faktorenanalytische Datenreduktion dieser sechs Mitarbeiter-Ratingskalen ergab eine Generalfaktorlösung mit 59\% Varianzaufklärung. Die Ladungen der Einzelskalen variierten zwischen 0,68 („Gesundheit“) und 0,86 („Persönlichkeitsstabilisierung“). Der Faktor wurde als „Therapieverbesserungen aus Mitarbeitersicht“ interpretiert. Die Faktorwerte wurden am Median ge- 
splittet, sodass zwei Teilgruppen mit relativ „größeren Therapieverbesserungen“ ( $n=76)$ bzw. mit "geringeren Therapieverbesserungen“ ( $n=75)$ aus Mitarbeitersicht operationalisiert wurden. Wegen fehlender Daten in einzelnen Ratingskalen lagen Faktorwerte für $\mathrm{n}=151$ vor.

Die vier Ergebniskriterien interkorrelierten zwischen 0,45 und 0,84 (Rangkorrelationen Spearman; alle $\mathrm{p}<0,0001$ ).

\section{Datenauswertung}

Die vorliegenden Daten wurden von einer wissenschaftlichen Hilfskraft in eine SPSS-Datenbank übertragen und einer systematischen Überprüfung und Fehlerbereinigung unterzogen, Umcodierungen sowie die Neuberechnung von Variablen wurden vorgenommen.

Bei der statistischen Auswertung der Daten wurden bei Nominal- oder Ordinalskalenqualität nichtparametrische Verfahren ( $\chi^{2}$-Test; Mann-Whitney-U-Test; Odds Ratio zur Angabe der Stärke des Gruppenunterschieds) angewandt, bei höherer Skalenqualität auch parametrische Tests (t-Test). Die multiple Regressionsanalyse wurde gerechnet bei intervallskalierter abhängiger Variablen, bei unabhängigen Variablen unterschiedlichen Skalenniveaus und dichotomen abhängigen Variablen die logistische Regression. Die Auswertung erfolgte mit dem Programm SPSS 10.0. Es wurde jeweils bei zweiseitiger Fragestellung als statistisches Entscheidungskriterium $\mathrm{p}<0,05$ gewählt.

\section{Ergebnisse}

\section{Deskription der Untersuchungsgruppe}

Die Untersuchungsgruppe war u.a. folgendermaßen näher charakterisiert:

Der Frauenanteil lag bei 26\%. Die Männer waren im Mittel 30,8 Jahre, die Frauen 27,8 Jahre alt $(\mathrm{p}<0,01)$; $30 \%$ waren 35 Jahre und älter, $8 \%$ jünger als 20 Jahre. $66 \%$ kamen aus Hamburg, $91 \%$ besaßen die deutsche Staatsangehörigkeit.

Die mittlere Dauer der Drogenabhängigkeit betrug 13,3 Jahre $(s=6,8) ; 83 \%$ hatten im letzten halben Jahr vor der Therapie polyvalent Drogen konsumiert, u.a. 76\% Heroin, $72 \%$ Kokain und $42 \%$ Benzodiazepine.

36\% hatten keinen Hauptschulabschluss, 77\% maximal Hauptschulabschluss. $72 \%$ wiesen keine abgeschlossene Berufsausbildung auf, und $48 \%$ hatten keine oder nur geringfügige Arbeitserfahrung. Im letzten halben Jahr vor der Therapie waren 76\% durchgängig weder im Arbeits- noch im Ausbildungsbereich integriert gewesen.

$85 \%$ waren vorbestraft; $54 \%$ begannen die Therapie mit einer Form juristischen Drucks (z. B. Bewährung; Weisung; §35 BtmG; ausstehendes Verfahren).

Im Mittel hatten die Aufgenommenen 5,7 ( $s=5,1)$ Krankenhausentzüge absolviert. Für $39 \%$ war es der erste stationäre Therapieversuch. $81 \%$ waren substituiert gewesen, $39 \%$ hatten an psychosozialer Betreuung neben der Substitution teilgenommen.
Im letzten halben Jahr vor der Therapie lebten $43 \%$ in eigener Wohnung, 20\% bei Angehörigen; die übrigen lebten in Institutionen oder waren wohnungslos.

$9 \%$ waren verheiratet, $13 \%$ geschieden und $78 \%$ ledig; $37 \%$ hatten mindestens ein Kind. Vor der Therapie lebten $45 \%$ alleinstehend und $42 \%$ in festen Beziehungen. $41 \%$ hatten im letzten halben Jahr vor der Therapie keine oder nur seltene Kontakte zu drogenfreien Personen.

$48 \%$ bezogen vor der Therapie Sozialhilfe, $11 \%$ hatten Einkommen aus Erwerbstätigkeit. 79\% waren verschuldet, 25\% mit Schulden von $10000 €$ und mehr.

\section{Belastende Lebensereignisse}

Tab.1 zeigt die Lebenszeitprävalenz verschiedener Lebensereignisse.

Tab. 1 Häufigkeit verschiedener belastender Lebensereignisse bei Teilnehmer/Innen einer stationären Drogentherapie $(n=188)$

\begin{tabular}{|c|c|c|c|c|}
\hline Lebensereignis & $\begin{array}{l}\text { gesamt } \\
n=188\end{array}$ & $\begin{array}{l}\text { Männer } \\
n=139\end{array}$ & $\begin{array}{l}\text { Frauen } \\
n=49\end{array}$ & Sign. \\
\hline Vater/Eltern unbekannt & $9 \%$ & $9 \%$ & $6 \%$ & \\
\hline $\begin{array}{l}\text { aufgewachsen ohne beide Eltern- } \\
\text { teile }\end{array}$ & $38 \%$ & $40 \%$ & $33 \%$ & \\
\hline Trennung der Eltern bis 16. Lj. & $29 \%$ & $30 \%$ & $25 \%$ & \\
\hline $\begin{array}{l}\text { mindestens ein Elternteil sucht- } \\
\text { mittelabhängig }\end{array}$ & $30 \%$ & $30 \%$ & $29 \%$ & \\
\hline $\begin{array}{l}\text { Heimaufenthalt/öffentliche Erzie- } \\
\text { hung }\end{array}$ & $29 \%$ & $27 \%$ & $33 \%$ & \\
\hline Tod enger Bezugspersonen & $18 \%$ & $16 \%$ & $22 \%$ & \\
\hline Psychiatrieerfahrung & $19 \%$ & $18 \%$ & $22 \%$ & \\
\hline $\begin{array}{l}\text { häufiger Wohnortwechsel in Kind- } \\
\text { heit/Jugend }\end{array}$ & $10 \%$ & $9 \%$ & $12 \%$ & \\
\hline $\begin{array}{l}\text { schwere körperliche Gewalterfah- } \\
\text { rung }\end{array}$ & $56 \%$ & $54 \%$ & $63 \%$ & \\
\hline sexuelle Gewalterfahrung & $21 \%$ & $9 \%$ & $53 \%$ & $p<0,0001$ \\
\hline Suizidversuch & $35 \%$ & $32 \%$ & $45 \%$ & \\
\hline Haftaufenthalt & $60 \%$ & $65 \%$ & $45 \%$ & $\mathrm{p}<0,05$ \\
\hline Ausübung schwerer Gewalttaten & $14 \%$ & $16 \%$ & $10 \%$ & \\
\hline Prostitution & $5 \%$ & $0 \%$ & $20 \%$ & $\mathrm{p}<0,0001$ \\
\hline $\begin{array}{l}\text { sonstiges stark belastendes } \\
\text { Ereignis }\end{array}$ & $28 \%$ & $21 \%$ & $47 \%$ & $p<0,0001$ \\
\hline
\end{tabular}

Die Frauen waren - verglichen mit den Männern - erheblich häufiger durch sexuelle Gewalterfahrungen ( $53 \%$ vs. $9 \%$ ) sowie durch (Straßen)prostitution (20\% vs. $0 \%$ ), ferner durch „sonstige stark belastende Ereignisse“ ( $47 \%$ vs. $21 \%$ ) belastet, die Männer waren häufiger inhaftiert gewesen (65\% vs. $45 \%$ ).

Bei 95\% war mindestens ein belastendes biographisches Ereignis dokumentiert, der Medianwert lag bei vier Ereignissen ( $M=4,0$; $s=2,4$; Range $0-10$ ).

Bei den Frauen lag die Life-time-Prävalenz im Mittel bei $\mathrm{M}=4,7$ $(s=2,2)$ und bei den Männern bei $M=3,8(s=2,2)$ belastenden Lebensereignissen (Mann-Whitney-U-Test; $z=2,05 ; \mathrm{p}<0,05$ ). 
Belastende Lebensereignisse und Therapieergebnisse Zusammenhang mit der Therapiezeit

Klient/Innen ohne dokumentierte schwere körperliche Gewalterfahrung hatten - verglichen mit Klient/Innen mit schwerer körperlicher Gewalterfahrung - eine im Mittel etwa um zwei Monate längere Therapiezeit. Ebenso hatten Klient/Innen ohne Hafterfahrungen bzw. ohne Suizidversuche relativ längere Therapiezeiten (Tab. $\mathbf{2}$ ).

Tab. 2 Belastende Lebensereignisse und Therapiezeit $(n=188)$

\begin{tabular}{|c|c|c|}
\hline & Therapietage & Sign. \\
\hline $\begin{array}{l}\text { mit schwerer körperlicher Gewalterfahrung } \\
(n=106)\end{array}$ & $\begin{array}{l}M=139 \\
s=121\end{array}$ & $\begin{array}{l}t=2,29 \\
d f=147,8\end{array}$ \\
\hline $\begin{array}{l}\text { ohne schwere körperliche Gewalterfahrung } \\
(\mathrm{n}=82)\end{array}$ & $\begin{array}{l}M=199 \\
S=118\end{array}$ & $p<0,001$ \\
\hline mit Hafterfahrung $(\mathrm{n}=112)$ & $\begin{array}{l}M=145 \\
s=115\end{array}$ & $\begin{array}{l}t=2,76 \\
d f=147,8\end{array}$ \\
\hline ohne Hafterfahrung $(n=76)$ & $\begin{array}{l}M=196 \\
s=130\end{array}$ & $p<0,007$ \\
\hline mit Suizidversuch $(n=66)$ & $\begin{array}{l}M=138 \\
s=125\end{array}$ & $\begin{array}{l}t=2,29 \\
d f=186\end{array}$ \\
\hline ohne Suizidversuch ( $n=122)$ & $\begin{array}{l}M=180 \\
s=120\end{array}$ & $p<0,023$ \\
\hline
\end{tabular}

Diese drei Merkmale wurden als „unabhängige Variablen“ in eine Regressionsanalyse mit der Therapiezeit (in Tagen) als abhängiger Variablen eingebracht. Es konnten insgesamt $8 \%$ der Therapiezeitvarianz erklärt werden $(R=0,30$; korrigiertes $\mathrm{R}^{2}=0,08$ ). Dabei wurden nur zwei signifikante Einflussvariablen identifiziert: schwere körperliche Gewalterfahrung als die relativ stärkere (Änderung in $R^{2}=0,05 ; p<0,001$ ), ferner noch Hafterfahrung (Änderung in $R^{2}=0,03 ; p<0,05$ ).

\section{Zusammenhang mit der Art der Therapiebeendigung (regulär vs. vorzeitig)}

Klient/Innen ohne schwere körperliche Gewalterfahrung beendeten häufiger die Therapie regulär (Tab. 3). Die Chancen dieser Klient/Innen, die Therapie regulär zu beenden, waren etwa dreimal so groß wie von Klient/Innen mit schweren körperlichen Gewalterfahrungen.

Tab. 3 Belastende Lebensereignisse und Art der Therapiebeendigung $(\mathrm{n}=188)$

\begin{tabular}{|c|c|c|}
\hline & $\begin{array}{l}\text { reguläre Therapie- } \\
\text { beendigung }\end{array}$ & Sign. \\
\hline $\begin{array}{l}\text { mit schwerer körperlicher Gewalt- } \\
\text { erfahrung }(n=106)\end{array}$ & $33 \%$ & $\begin{array}{l}\chi^{2}=14,59 \\
\mathrm{df}=1\end{array}$ \\
\hline $\begin{array}{l}\text { ohne schwere körperliche Gewalt- } \\
\text { erfahrung }(n=82)\end{array}$ & $61 \%$ & $\begin{array}{l}\mathrm{P}<0,0001 \\
\mathrm{OR}=3,1\end{array}$ \\
\hline mit Hafterfahrung ( $n=112)$ & $38 \%$ & $\begin{array}{l}\chi^{2}=6,65 \\
\mathrm{df}=1\end{array}$ \\
\hline ohne Hafterfahrung $(\mathrm{n}=76)$ & $57 \%$ & $\begin{array}{l}\mathrm{P}<0,05 \\
\mathrm{OR}=2,2\end{array}$ \\
\hline mit Suizidversuch $(n=66)$ & $35 \%$ & $\begin{array}{l}\chi^{2}=4,41 \\
\mathrm{df}=1\end{array}$ \\
\hline ohne Suizidversuch ( $n=122$ ) & $51 \%$ & $\begin{array}{l}\mathrm{P}<0,05 \\
\mathrm{OR}=2,0\end{array}$ \\
\hline
\end{tabular}

Ebenso beendeten Klient/Innen ohne Hafterfahrungen bzw. ohne Suizidversuch häufiger regulär die Therapie, und zwar jeweils etwa mit der zweifachen relativen Chance.

In eine logistische Regression (Methode: vorwärts schrittweise) wurden die Merkmale schwere körperliche Gewalterfahrung, Hafterfahrung und Suizidversuch als unabhängige Prädiktoren mit der Art der Therapiebeendigung (regulär vs. vorzeitig) als abhängige Variable eingebracht. Ausschließlich das Merkmal schwere körperliche Gewalterfahrung verbesserte die Vorhersage ( $B=1,15$; Wald $=14,17 ; \mathrm{df}=1 ; \mathrm{p}<0,0001)$. Das Modell erklärte etwa $13 \%$ der Varianz (Nagelkerkes $R^{2}=0,134$ ). Insgesamt wurde bei 64\% der Fälle die Art der Therapiebeendigung richtig vorhergesagt (vorzeitig: 69\% regulär: 59\%).

\section{Zusammenhang mit der Integration in Arbeit/Ausbildung bei Therapiebeendigung (integriert vs. nicht-integriert)}

Klient/Innen ohne schwere körperliche Gewalterfahrungen waren am Therapieende häufiger in Arbeit oder Ausbildung integriert ( $56 \%$ vs. $39 \% \chi^{2}=4,73 ; \mathrm{df}=1 ; \mathrm{p}<0,05$ ). Die Chancen der beruflich-schulischen Integration waren gegenüber jenen mit schweren körperlichen Gewalterfahrungen zweimal so hoch $(\mathrm{OR}=2,0)$

\section{Zusammenhang mit Therapieverbesserungen aus Mitarbeitersicht (größere vs. geringere)}

Klient/Innen ohne schwere körperliche Gewalterfahrungen hatten häufiger größere, über dem Median liegende Therapieverbesserungen aus Mitarbeitersicht erzielt ( $65 \%$ vs. $38 \% ; \chi^{2}=4,73$; $\mathrm{df}=1 ; \mathrm{p}<0,001 ; \mathrm{OR}=3,1)$.

Alle anderen belastenden Lebensereignisse standen jeweils in keinem signifikanten Zusammenhang mit den Ergebniskriterien. Unter Anwendung der Bonferroni-Korrektur für multiple Signifikanztests war nur der Zusammenhang zwischen schweren körperlichen Gewalterfahrungen und der Art der Therapiebeendigung (regulär vs. vorzeitig) signifikant $(p<0,05)$, tendenziell noch mit der Therapiezeit und den Therapieverbesserungen aus Mitarbeitersicht $(\mathrm{p}<0,10)$.

\section{Extremgruppenvergleiche}

Durch Unterteilung am Medianwert wurden zwei Extremgruppen mit relativ weniger $(M=1,8 ; s=1,0$; Range $0-3 ; n=83)$ und relativ mehr belastenden Lebensereignissen $(M=5,6 ; s=1,6$; Range 4-10; $\mathrm{n}=105$ ) gebildet.

Es zeigte sich, dass die Klient/Innen mit weniger biographischen Belastungen eine längere Therapiezeit hatten und die Therapie häufiger regulär beendet hatten; sie waren am Therapieende häufiger beruflich-schulisch integriert, und gemäß Mitarbeiterrating hatten sie häufiger größere Therapieverbesserungen in verschiedenen Bereichen erzielt (Tab. 4).

Es gab keine signifikanten geschlechtspezifisch unterschiedlichen Zusammenhänge zwischen belastenden Lebensereignissen und den berücksichtigten Therapieergebniskriterien. 


\begin{tabular}{|c|c|c|c|}
\hline & $\begin{array}{l}\text { weniger belastende } \\
\text { Lebensereignisse }\end{array}$ & $\begin{array}{l}\text { mehr belastende } \\
\text { Lebensereignisse }\end{array}$ & Sign. \\
\hline $\begin{array}{l}\text { Therapiezeit } \\
(n=188)\end{array}$ & $\begin{array}{l}M=191 \\
s=119\end{array}$ & $\begin{array}{l}M=145 \\
s=123\end{array}$ & $\begin{array}{l}t=2,57 ; d f=186 \\
p<0,011\end{array}$ \\
\hline $\begin{array}{l}\text { reguläre Therapiebeendigung } \\
(n=188)\end{array}$ & $54 \%$ & $38 \%$ & $\begin{array}{l}\chi^{2}=4,86 ; d f=1 \\
p<0,027 ; O R=1,9\end{array}$ \\
\hline $\begin{array}{l}\text { Integration in Arbeit/Ausbildung } \\
(n=163)\end{array}$ & $55 \%$ & $39 \%$ & $\begin{array}{l}\chi^{2}=4,11 ; d f=1 ; \\
p<0,043 ; O R=1,9\end{array}$ \\
\hline $\begin{array}{l}\text { größere Therapieverbesserun- } \\
\text { gen aus Mitarbeitersicht } \\
(n=151)\end{array}$ & $62 \%$ & $41 \%$ & $\begin{array}{l}\chi^{2}=6,47 ; d f=1 ; \\
p<0,011 ; O R=2,4\end{array}$ \\
\hline
\end{tabular}

Tab. 4 Therapieergebnisse von Klient/Innen mit mehr bzw. weniger belastenden Lebensereignissen

\section{Diskussion}

Die Ergebnisse zeigen die insgesamt außerordentlich hohe Belastung der Therapieteilnehmer/Innen durch eine Vielzahl belastender Lebenserfahrungen. Dabei muss angenommen werden, dass es sich bei den berichteten Prävalenzwerten um Mindestwerte handelt, da weitere kritische Life-events vermutlich in der Therapie nicht thematisiert oder nicht dokumentiert worden sind. Die Anteile von Klient/Innen mit körperlicher oder sexueller Gewalterfahrung, Haft- bzw. Heimaufenthalten lagen in etwa auf dem Niveau, das im gleichen Zeitraum in der Hamburger Basisdatendokumentation methodisch auf andere Weise erhoben wurde [24-25]. Dies kann als Hinweis auf die Validität der über die Auswertung der Therapiedokumentationen erhobenen Daten betrachtet werden, obwohl das Fehlen von Reliabilitätsangaben eine methodische Schwäche der vorliegenden Studie ist.

Frauen hatten häufiger sexuelle Gewalt erfahren, sie beschafften ihr Geld häufiger über Prostitution, und sie waren etwas seltener inhaftiert. Diese Befunde ordnen sich in das vorliegende Wissen ein, interessant ist jedoch die erheblich häufigere Belastung von Frauen durch ein „sonstiges belastendes Ereignis“. In dieser Kategorie waren Einzelerlebnisse - u. a. Miterleben schwerer Unfälle, von schweren Gewalttaten, schwerer eigener Unfall, lebensbedrohliche Erkrankung - zusammengefasst worden, die eine zur statistischen Auswertung zu geringe Einzelhäufigkeit hatten. Es deuteten sich weitere geschlechtsspezifische Unterschiede an, die jedoch nicht signifikant waren. Insgesamt waren die Frauen biographisch durch etwas mehr Lebensereignisse belastet.

Es gab nur wenige Hinweise, dass einzelne belastende Lebensereignisse einhergehen mit schlechteren Therapieergebnissen. Dabei kristallisierte sich heraus, wenn man das für multiple Tests korrigierte Signifikanzniveau berücksichtigt, aber auch nach den multivariaten Analysen, dass Klient/Innen mit schweren körperlichen Gewalterfahrungen schlechtere Therapieergebnisse hatten. Sie beendeten die Therapie häufiger vorzeitig durch „Abbruch“ oder Entlassung, ihre Therapiezeit war entsprechend im Mittel etwa zwei Monate kürzer. Gemäß Mitarbeitereinschätzung hatten sie seltener größere, operationalisiert als über dem Median liegende Verbesserungen in verschiedenen Bereichen in der Therapie erzielt. Die genauere Analyse zeigte jedoch, dass mit $8 \%$ bzw. $13 \%$ in den Regressionsanalysen nur ein begrenzter Teil der Varianz der jeweiligen Ergebniskriterien aufgeklärt werden konnte. Andererseits: Es darf vermutet werden, dass bei einigen derjenigen, die der Teilgruppe ohne schwere körperliche Gewalterfahrungen zugeordnet wurden, tatsächlich solche vor- lagen (s.o.), sodass ggf. beim Vergleich von tatsächlich unterschiedlichen Gruppen der Effekt stärker ausgefallen wäre.

Darauf hinzuweisen ist, dass das Vorliegen sexueller Gewalterfahrungen nicht mit den verschiedenen Therapieergebnissen zusammenhing, auch nicht, wenn geschlechtsspezifisch ausgewertet wurde. Das mag ebenfalls mit der geringen Varianz zwischen den Teilgruppen zusammenhängen, wenn es bei den Frauen ohne sexuelle Gewalterfahrungen tatsächlich eine größere Dunkelziffer gegeben hat. Vielleicht spiegelt das Ergebnis auch die in der Therapie erfolgte individuelle Förderung und Unterstützung wider, sodass unterschiedliche Belastungen in der Therapie ausgeglichen werden. In ähnliche Richtung gehen Ergebnisse, dass Klient/Innen mit Gewalterfahrungen in der ambulanten Betreuung zumindest keine kürzeren Betreuungszeiten bzw. dementsprechend keine höheren Abbruchquoten hatten [30]. In einer stationären Entwöhnungseinrichtung standen Merkmale psychiatrischer Komorbidität, die oftmals als Folge schwer belastender Lebenserfahrungen entstanden, ebenfalls nicht in Zusammenhang mit dem Therapieabbruch [28].

Beim Vergleich von zwei Extremgruppen mit relativ vielen und weniger belastenden Lebensereignissen konnten im Sinne der Eingangshypothese Unterschiede in allen Therapieergebniskriterien festgestellt werden: Die relativ weniger belasteten Therapieteilnehmer/Innen beendeten die Therapie häufiger regulär, ihre Therapiezeit war länger. Sie waren am Therapieende häufiger beruflich-schulisch integriert, und sie hatten häufiger größere Therapieverbesserungen gemäß Mitarbeitereinschätzung erzielt. Die Chancen dieser Therapieteilnehmer/Innen, bessere Therapieergebnisse zu erzielen, waren etwa zweimal höher, verglichen mit den Klient/Innen mit überdurchschnittlich vielen biographischen Belastungen.

Die vorliegenden Ergebnisse müssen mit der Einschränkung gesehen werden, dass sie in nur einer Einrichtung mit einem spezifischen konzeptionellen Ansatz und insgesamt erheblich und überdurchschnittlich psychosozial belasteten Therapieteilnehmer/Innen gefunden wurden. Dadurch war die Varianz in der Untersuchungsgruppe eingeschränkt. Auch ist selbstverständlich nichts über Kausalbeziehungen, moderierende Variablen und vor allem Therapieprozesse und entsprechende Wechselwirkungen ausgesagt.

Letztlich kann jedoch die Hypothese formuliert werden, dass die Häufung belastender Lebensereignisse in der Biographie Drogenabhängiger mit schlechteren Therapieergebnissen in der statio- 
nären Drogentherapie einhergeht. Bei Betrachtung von Einzelereignissen lässt das Vorliegen schwerer körperlicher Gewalterfahrungen schlechtere Therapieergebnisse vermuten. $\mathrm{Ob}$ durch eine erhöhte Sensibilität für den besonderen Hilfebedarf der besonders stark belasteten Klient/Innen, mehr Therapieeinheiten [23] und zusätzliches Integrieren [22, 26, 27] von spezifischen Hilfsangeboten auch die stationären Rehabilitationsergebnisse verbessert werden können, bleibt zu überprüfen.

\section{Literatur}

${ }^{1}$ Kuhn S. Trauma, Posttraumatische Belastungsstörung und Substanzabhängigkeit: Eine Literaturübersicht. Suchttherapie 2004; 3: $110-117$

${ }^{2}$ Schäfer M, Schnack B, Soyka M. Sexueller und körperlicher Missbrauch während früher Kindheit oder Adoleszenz bei späterer Drogenabhängigkeit. Psychother Psychosom med Psychol 2000; 50: $38-50$

${ }^{3}$ Krausz M, Briken P. Sexueller Missbrauch bei opiatabhängigen Frauen in Relation zu biografischen Faktoren, Suchtentwicklung und psychischer Symptomatik. Suchttherapie 2002; 3: 178-183

${ }^{4}$ Haas S, Enders-Dragässer U. Sucht als Über-Lebenschance für Frauen mit Gewalterfahrung. Wiener Zeitschrift für Suchtforschung 2003; 3/4: $37-48$

${ }^{5}$ Zenker C, Bammann K, Jahn I. Ursachen und Differenzierung der Abhängigkeitserkrankungen bei Frauen. Sucht aktuell 2003; 2: 15-20

${ }^{6}$ Thiel G, Ackermann R, Prinzleve M. Rehabilitationsergebnisse von 766 Therapiebeendern in den Hamburger Sozialtherapeutischen Wohngemeinschaften für Drogenabhängige. Suchttherapie 2004; 5: $180-190$

${ }^{7}$ Kemner C, Klein M, Zemlin U. Gewalterfahrungen bei Patientinnen und Patienten einer Fachklinik für Alkoholabhängige: Ergebnisse einer Prävalenzerhebung und Konsequenzen für das Behandlungsprogramm. Suchttherapie 2004; 3: $109-160$

8 Beg D, Quinten C. Gewalterfahrung von SuchtpatientInnen - Eine empirische Untersuchung zum Ausmaß und zu den wahrgenommenen psychischen Folgen zurückliegender Gewalterfahrungen. Praxis Klinische Verhaltensmedizin und Rehabilitation 2000; 50: 86-92

${ }^{9}$ Neumann E, Martens MS, Buth S. 2004. Ambulante Suchthilfe in Hamburg. Statusbericht der Hamburger Basisdatendokumentation. In: BADO e.V. (Hrsg). Hamburg: Kreutzfeldt, 2005

${ }^{10}$ Ouimette PC, Finney JW. Two-year posttreatment functioning and coping of substance abuse patients with posttraumatic stress disorder. Psychology of Addictive Behaviors 1999; 13: 105 - 114

${ }^{11}$ Ouimette PC, Brown PJ. Trauma and substance abuse. Causes, consequences, and treatment of comorbid disorders. Washington, DC: American Psychological Association, 2003

12 Ouimette PC, Ahrens C, Moos RH et al. Posttraumatic stress disorder in substance abuse patients: Relationship to 1-year posttreatment outcomes. Psychology Addict Behaviors 1997; 11: 34-47

${ }^{13}$ Hien DA, Nunes E, Levin FR et al. Posttraumatic stress disorder and short-term outcome in early methadone treatment. Journal of Substance Abuse Treatment 2000; 19 (1): 31 - 37
${ }^{14}$ Najavits L, Weiss R, Shaw S. A clinical profile of women with posttraumatic stress disorder and substance dependence. Psychology of Addictive Behaviors 1999; 13: 98 - 104

15 Ouimette PC, Moos RH, Finney JW. Two-year mental health service use and course of remission in patients with substance use and posttraumatic stress disorders. Journal of Studies on Alcohol 2000; 61 (2): $247-253$

${ }^{16}$ Brady KT, Killeen TK, Saladin ME et al. Comorbid substance abuse and posttraumatic stress disorder: Characteristics of women in treatment. American Journal on Addictions 1994; 3 (2): 160 - 164

${ }^{17}$ Najavits L. Clinicians' views on treating posttraumatic stress disorder and substance use disorder. Journal of Substance Abuse Treatment 2002; 22: 79-85

18 Teegen F, Zumbeck S. Prävalenz traumatischer Erfahrungen und Posttraumatischer Belastungsstörung bei substanzabhängigen Personen. Psychotherapeut 2000; 45: 44-49

${ }^{19}$ Schäfer I, Schultz M, Verthein U et al. Traumatisierungen bei Suchtpatient(inn)en - Relevanz und spezifische Behandlung in der ambulanten Suchttherapie. Suchttherapie 2004; 5: 118-123

${ }^{20}$ Jaycox LH, Ebener P, Damesek L et al. Trauma exposure and retention in adolescent substance abuse treatment. Journal of tramatic stress 2004; 17 (2): $113-121$

${ }^{21}$ Ouimette PC, Brown PJ, Najavits LM. Course and treatment of patients with both substance use and posttraumatic stress disorders. Addictive behaviors 1998; 23 (6): $785-795$

22 Ouimette PC, Ahrens C, Moos RH et al. During treatment changes in substance abuse patients with posttraumatic stress disorder. The influence of specific interventions and program environments. Journal of substance abuse treatment 1998; 15 (6): 555 -564

${ }^{23}$ Rosen CS, Ouimette PC, Sheikh JI et al. Physical and sexual abuse history and addiction treatment outcomes. Journal of studies on alcohol 2002; 63 (6): 683-687

${ }^{24}$ Martens SM, Lorenzen J, Verthein U et al. 2002. Ambulante Suchthilfe in Hamburg. Statusbericht der Hamburger Basisdatendokumentation. In: BADO e. V. (Hrsg). Hamburg: Kreutzfeldt, 2003

${ }^{25}$ Martens SM, Degkwitz P, Richter E et al. 2003. Ambulante Suchthilfe in Hamburg. Statusbericht der Hamburger Basisdatendokumentation. In: BADO e.V. (Hrsg). Hamburg: Kreutzfeldt, 2004

${ }^{26}$ Morrissey JP, Jackson EW, Ellis AR et al. Twelve-month outcomes of trauma-informed interventions for women with co-occuring disorders. Psychiatric services: a journal of the American Psychiatric Association 2005; 56 (10): $1213-1222$

${ }^{27}$ Ouimette PC, Moos RH, Finney JW. PTSD treatment and 5-year remission among patients with substance use and posttraumatic stress disorders. Journal of consulting and clinical psychology 2003; 71 (2): $410-414$

${ }^{28}$ Günthner A, Dedner C, Schäfer G et al. Komorbidität bei Drogenabhängigen - Empirische Ergebnisse und therapeutische Konsequenzen. Suchttherapie 2000; 1 (1): 16 -20

${ }^{29}$ Witfoot J, Driessen M. Alkoholabhängigkeit und psychiatrische Komorbidität - ein Überblick. Suchttherapie 2000; 1 (1): 8-15

${ }^{30}$ Lorenzen J, Rebernig E, Martens MS. Gewalterfahrungen in der Lebensgeschichte von Klient(inn)en der Hamburger ambulanten Suchthilfe. Suchtmedizin 2005; 7 (2): 148 